\title{
AN EXPERIMENTAL STUDY ON AN INVERTEBRATE (Isonychia japonica) RESPONSE TO APPROACH FLOW VELOCITY AND BLOCKS AS REFUGIA
}

\author{
Prem Shah ${ }^{1}$, Norio Tanaka ${ }^{2}, *$ and Eiichi Furusato ${ }^{3}$ \\ ${ }^{1}$ Member of JSCE, Graduate School of Science and Engineering, Saitama University (255 Shimo-Okubo, Sakura-ku, \\ Saitama-shi, 338-8570, Saitama, Japan) \\ ${ }^{2}$ Member of JSCE, *Corresponding author, Dr. of Eng., Professor, Graduate School of Science and Engineering, \\ Saitama University, (255 Shimo-Okubo, Sakura-ku, Saitama-shi, 338-8570, Saitama, Japan), \\ email:tanaka01@mail.saitama-u.ac.jp \\ ${ }^{3}$ Member of JSCE, Dr. of Eng., Assistant Professor, Graduate School of Science and Engineering, Saitama \\ University (255 Shimo-Okubo, Sakura-ku, Saitama-shi, 338-8570, Saitama, Japan)
}

\begin{abstract}
Behavior of an invertebrate, Isonychia japonica, was investigated based on their response to approach flow velocity. Without refugia, the behavior has been categorized into four, walking or crawling, passive walking (or no walking), washout and enduring behavior. The critical depth-averaged velocity for no walking is $0.2 \mathrm{~m} / \mathrm{s}$ and for washout is $0.4 \mathrm{~m} / \mathrm{s}$ for the specie. The corresponding local velocity calculated from PIV analysis at the invertebrate height for no walking and washout is 0.12 and $0.22 \mathrm{~m} / \mathrm{s}$, respectively. The blocks were placed as refugia for the invertebrates and were successful in providing refugia to the invertebrates. With blocks as refugia, washout behavior was not observed. The local velocity around invertebrate habitat after placement of blocks was reduced to less than $0.1 \mathrm{~m} / \mathrm{s}$ during high flow conditions which is less than the critical velocity conditions without refugia.
\end{abstract}

Key Words: Invertebrates behavior, Isonychia japonica, flow response, refugia

\section{INTRODUCTION}

There is a need for more information on the species-specific movements and behavior of benthic invertebrates in response to changes in near-bed hydraulics (change in flow condition) ${ }^{1)}$. The basic movement behavior or characteristics of invertebrates have been already explained ${ }^{2)}$. However, in a stream, invertebrates are exposed to several flow conditions, and its movement behavior will be influenced by the flow velocity.

During high flow or flood conditions, the basic need for refugia increases and invertebrates are forced to seek some kind of refuge. Matthaei et al. ${ }^{3)}$ investigated about the importance of stable stones for invertebrate refugia during the floods Other researchers for e.g. Lancaster ${ }^{4}$, Cobb et al. ${ }^{5)}$ have also explained the importance of refugia or microhabitat preference based on abundance near refugia. Although this information is useful for restoration projects, there exists no information on the hydraulic parameter such as invertebrate's velocity preference around their body height near refugia.

In this research, Isonychia japonica was chosen for the simple reason that they are one of the invertebrates that needs refugia during flood conditions because their natural habitat is near the stream rock bed. Furthermore, from the past research $^{6}$ Isonychia japonica were found to be swimming and clinger type invertebrates, and the basic behavior could be further classified based on the flow condition ${ }^{6}$. Large bed material has higher function of providing refugia. However, the blocks (including large rocks) and its arrangement can be used as refugia for invertebrates during underscour condition also. The information on block's arrangement and design could be helpful for invertebrate restoration projects.

So, the objective of this study is (a) to define 
critical velocity for walking and washout or dislodgement under no refugia condition, and (b) to provide suitable refugia for invertebrate and understand the importance of refugia in reducing the flow velocity near its habitat.

\section{METHODOLOGY}

\section{(1) Experimental condition and flume setup}

The flume size of $25 \mathrm{~cm}$ wide $(W), 20 \mathrm{~cm}$ deep $(D)$ and $180 \mathrm{~cm}$ long $(L)$ was used for the experiment (Figs. 1, $2 \&$ 3). The experiment was conducted for a range of flow velocities in Table 1. Experiment was conducted for invertebrates without any refugia (Case I) and invertebrates with block as refugia (Case II). Wooden bed surface with a Manning roughness coefficient $(n)$ of 0.013 was used. Invertebrate movement was observed under different flow conditions. For each flow condition, velocity $\left(U_{a}\right)$ was measured at upstream (location: $X_{0}$ for Case I and $X_{I}$ for Case II) (refer Fig. 3) and averaged along the depth.

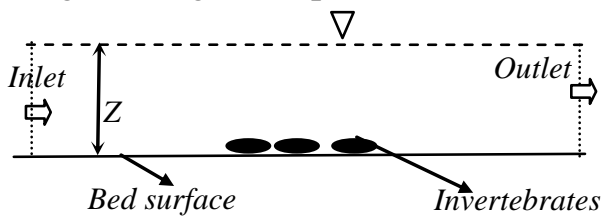

Fig.1 Schematic view of the flume with invertebrates without refugia (side view); Case I

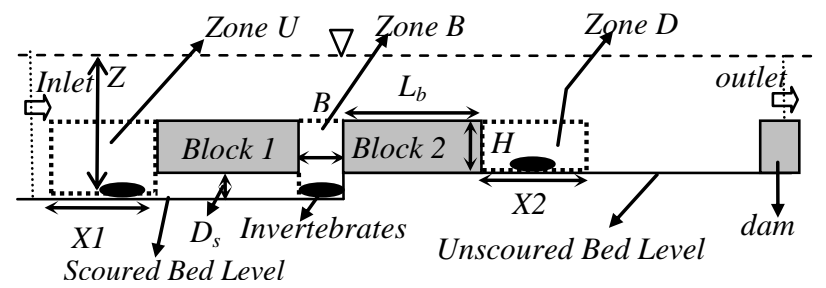

Fig.2 Arrangement of two blocks for refugia, initial location of invertebrates, and the definition of different characteristic zone (side view); Case II

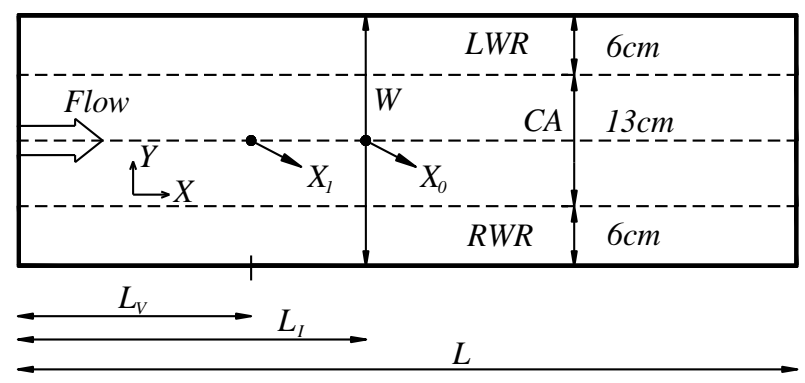

Fig.3 Definition of Left Wall Region ( $L W R)$, Central Area (CA) and Right Wall Region $(R W R)$ in the flume (plan view): $L$ is the length of the flume; $W$ is the width of the flume; $X_{0}$ is the initial position of invertebrate placement and the location of approach flow velocity measurement at a distance $L_{I}=90 \mathrm{~cm}$ from upstream for Case I, $X_{I}$ is the location of approach flow velocity measurement location for Case II at a distance $L_{V}=70 \mathrm{~cm}$ from upstream.
Table 1 Hydraulic condition and number of invertebrate used for the experiment

\begin{tabular}{|c|c|c|c|c|}
\hline & No refugia & \multicolumn{3}{|c|}{ Refugia } \\
\hline \hline Cases & Case I & \multicolumn{3}{|c|}{ Case II } \\
\hline Depth (m) & $0-0.05$ & \multicolumn{3}{|c|}{$0-0.10$} \\
\hline Velocity (m/s) & $0-0.52$ & \multicolumn{3}{|c|}{$0-0.50$} \\
\hline $\begin{array}{c}\text { Number of the } \\
\text { invertebrates } \\
\text { used } \\
\text { (individuals) }\end{array}$ & 16 & 7 & 7 & 7 \\
\cline { 3 - 5 } & & Zone $U$ & Zone $B$ & Zone $D$ \\
\hline
\end{tabular}

Table 2 Total flow conditions for each observation (unit: cases)

\begin{tabular}{|c|c|c|}
\hline & No refugia & Refugia \\
\hline \hline Cases & Case I & Case II \\
\hline $1^{\text {st }}$ observation & 18 & 10 \\
\hline $2^{\text {nd }}$ observation & 18 & 12 \\
\hline $3^{\text {rd }}$ observation & 16 & 12 \\
\hline
\end{tabular}

For refugia, a good arrangement was required, which can simulate the natural habitat of invertebrates in a stream. For that purpose, two wooden blocks of height $(H=20 \mathrm{~mm})$ and length $\left(L_{b}\right.$ $=8 \mathrm{~cm})$ were put inside the flume with first block having (Block1) a shallow underscour depth $\left(D_{s} / H\right.$ of 0.05 , where, $D_{s}$ is the underscour depth) and second block (Block2) fixed to the bed surface (Fig. 2). The simple setup of shallow scouring depth (1mm) less than invertebrate body height $(4 \mathrm{~mm})$ also removed possibility that individuals could take refuge underneath block. A small gap $(B)$ of $20 \mathrm{~mm}$ (equal to body length of invertebrates) was placed between Block1 and Block2. At downstream of Block2, a small dam was constructed at the exit of the flume to create a pool. Zone $U$ is defined as the upstream region of Block1 with distance $X 1$, Zone $B$ is defined as the region between Block1 and Block2 with horizontal distance $B$, and Zone $D$ is defined the region downstream of Block2 with horizontal distance $X 2$ (Fig.2).

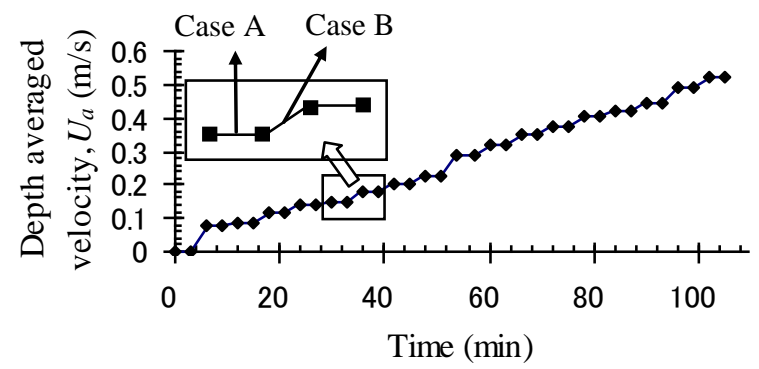

Fig.4 Example of velocity increment with time (Case I, $1^{\text {st }}$ observation): Case A represents uniform flow stage and Case B represents incremental flow stage 
We conducted the experiment for Case I and Case II with three observations as shown in Table 2 and Fig. 4 shows the velocity increment with time for the Case I, $1^{\text {st }}$ observation. The invertebrates were put in the flume, and the velocity was gradually increased. The velocity was increased gently for 3 minutes till the water inside the flume channel became uniform. When the velocity was uniform, the invertebrates were observed for 3 minutes (Case A), and their response to velocity increment was investigated. However, during velocity incremental stage invertebrate's movements were also observed (Case B).

\section{(2) Invertebrate type and its behavior}

Invertebrate used in this experiment is Isonychia japonica species from family Emphemerella. They were collected from a stream in Fukushima Prefecture and were kept inside the aquarium during the experiment. Every week, a fresh batch of invertebrates was collected and was added inside the aquarium. The body length (with tail) of the invertebrate is $20 \mathrm{~mm}$ (average) with a standard deviation of $2.35 \mathrm{~mm}$ and the body height is $4 \mathrm{~mm}$ (average). For every experiment, 16-21 insects were taken out of the aquarium and after the experiment, they were put back inside the aquarium. The tap water used in the flume channel and aquarium was dechlorinated. The weaker invertebrates were not used in the experiment. The water temperatures during the experiments ranged from 15 to $20^{\circ} \mathrm{C}$ both in flume channel and aquarium.

From the preliminary investigation, the observed invertebrate behavior can be classified into four; 1) active walking, 2) passive walking or no walking, 3) enduring behavior, and 4) washout. Active walking is defined as the active movement of invertebrates in search for refugia. This type of walking includes movement along the width of the flume channel (for e.g. left wall to center, center to right wall, center to left wall and right wall to center inside the flume channel). To define the position of invertebrates inside the flume as right wall or center or left wall, the whole flume was divided into three regions. Right wall region (RWR) is defined as the $6 \mathrm{~cm}$ distance from the right wall. Likewise, left wall region (LWR) is defined as the $6 \mathrm{~cm}$ distance from the left wall. The rest $13 \mathrm{~cm}$ is defined as the central area (CA) as shown in Fig. 3. The number of invertebrates showing active walking behavior was calculated by counting the number of invertebrates moving from their position (i.e. centre to left wall or vice versa).

Passive walking or no walking is defined as the movement of invertebrates upon physical disturbances such as attack of prey or human interferences. In this type of behavior, invertebrates don't change their position. Enduring behavior is defined as the adjustment of invertebrate body position and no movement of invertebrate upon the change in flow condition. It is difficult to differentiate passive walking or no walking behavior from enduring behavior only from observation. In this experiment, physical disturbance (carefully touch invertebrates by a thin and long stick to check whether they move or not) was applied to check the behavior of invertebrate. When they were touched by a thin stick, the flow pattern was not disturbed. Washout is defined as the detachment or dislodgement of invertebrate from its original position by fluid force or exit out of the flume channel.

\section{(3) Particle Image Velocimetry (PIV)}

Aluminum particles were used as tracers for visualizing flow. The green laser (Green Laser Sheet of $200 \mathrm{~m} / \mathrm{G}$, Katoh Koken Co., Ltd.) was mounted at the top of the flume. A high-speed digital camera (K-II, Katoh Koken TV Zoom lens H851V, Katoh Koken Co., Ltd.) was placed near the flume wall. For no block case (Case I), the video was taken at the halfway length of the flume channel and the center of the channel. For block case (Case II), the video was taken upstream of Block1 (Zone $U$ ), inside the gap between Block1 and Block2 (Zone $B$ ) and downstream of Block2 (Zone $D$ ) as shown in Fig. 2

\section{OBSERVATION AND ANALYSIS OF INVERTERBATE BEHAVIOR}

\section{(1) Invertebrate response to change in hydraulic condition (velocity) (Case I) \\ Fig. 5 shows the walking behavior of} invertebrates for different depth averaged velocity. Initially, invertebrates were put inside the flume channel, and flow was directed from upstream towards the invertebrates. Even before the flow started $\left(U_{a}\right.$ of $\left.0 \mathrm{~m} / \mathrm{s}\right)$, many number of invertebrates were walking i.e. $\left(5,3\right.$ and 8 individuals in $1^{\text {st }}, 2^{\text {nd }}$ and $3^{\text {rd }}$ observations respectively) (Fig.3). When the flow started, invertebrates showed a quick response to the flow by walking to avoid the flow. As a result number of invertebrate that show walking behavior is up to 6 individuals at $U_{a}$ of $0.1 \mathrm{~m} / \mathrm{s}$. Invertebrates tired to endure the flow and started to find some comfortable places inside the flume. The most convenient place inside the flume without any refugia is the side wall region (Fig. 3). So, when the flow started, the walking behavior of invertebrates was highly active. As the depth averaged velocity $\left(U_{a}\right)$ gradually increased, the number of walking 
invertebrates was reduced as shown in Fig. 5. The active walking was observed when $U_{a}$ was $0-0.2 \mathrm{~m} / \mathrm{s}$. It was also noted that even for depth averaged velocity range of $0-0.2 \mathrm{~m} / \mathrm{s}$, the invertebrates mostly showed a high response to flow during incremental stage of velocity (Case B). It implies that when velocity is increased, the invertebrates that are already comfortable around their present location have to adjust to the new flow velocity. Invertebrates can do the adjustment by either making its body streamlined to flow or moving to another safer location. When the $U_{a}$ reached $0.2 \mathrm{~m} / \mathrm{s}$, most of the invertebrates were at rest. We can assume that by this time, the invertebrates have already found the best possible position inside the flume because invertebrates completely stopped showing active walking behavior after $U_{a}$ of 0.2 $\mathrm{m} / \mathrm{s}$. This, $U_{a}$ of 0.2 , is the critical depth average velocity value for no walking. We can see in Fig.5 that passive walking or no walking could be seen from $U_{a}$ of $0.2-0.4 \mathrm{~m} / \mathrm{s}$.

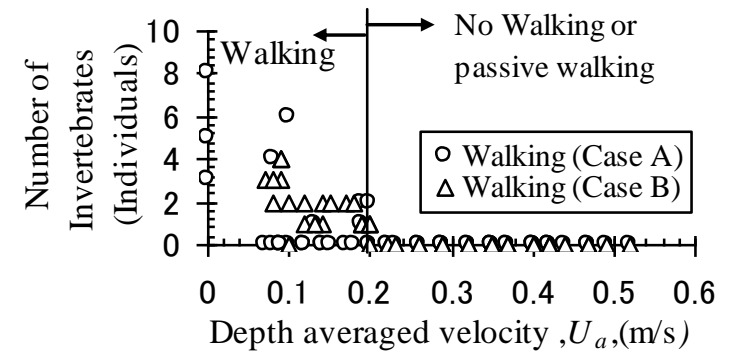

Fig. 5 Critical value of depth-averaged velocity for no walking (Case I) (See Fig.4, Table 1 and Table 2)

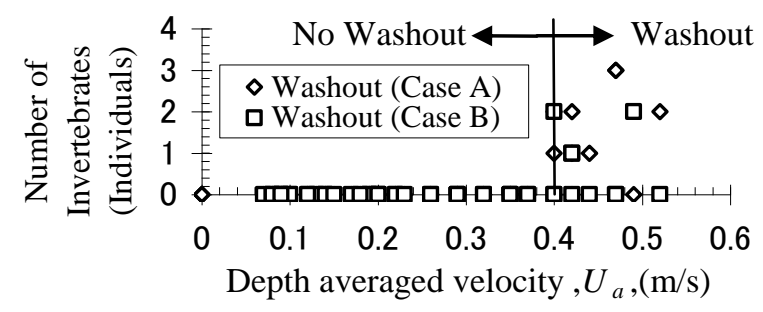

Fig.6 Critical value of depth-averaged velocity for washout (Case I) (See Fig.4, Table 1 and Table 2)

We can see in Fig. 6 that from $U_{a}$ of $0-0.4 \mathrm{~m} / \mathrm{s}$, invertebrates were not washed out and after $U_{a}$ of $0.4 \mathrm{~m} / \mathrm{s}, 1$ and 2 number of invertebrates was washed out from the flume channel for uniform flow condition (Case A) and change in flow condition (Case B) respectively. This, $U_{a}$ of 0.4 , is the critical depth average velocity value for washout. At this stage, they need some kind of refuge because their best selected area is not good.

(2) Invertebrate response to change in hydraulic condition (velocity) for blocks as refugia (CaseII)

Fig.7 shows the number of invertebrates walking with refugia for a depth averaged velocity $\left(U_{a}\right)$ during three observations. At $U_{a}$ of $0 \mathrm{~m} / \mathrm{s}$ (stagnant water condition), invertebrates (1 individual) showed walking behavior at Zone $U$, Zone $B$ and Zone $D$ at one of the observations (out of 3 observations). However, in other two observations, they did not show any walking movement before starting of flow. Soon after the flow was introduced inside the flume, more number of invertebrates started to show active walking behavior to find refugia. At Zone $U$, block's front face as well as the side wall was used for refugia by invertebrates. At Zone $B$, the invertebrates preferred the central area (CA) than wall area (refer Fig. 3 for the definition of $\mathrm{CA}$ ). Invertebrates actively walked for both Case A (uniform velocity stage) and Case B (velocity incremental stage).

At Zone $U$ and Zone $B$, the active walking behavior was observed until the depth average velocity, $U_{a}$, of $0.35 \mathrm{~m} / \mathrm{s}$ (Fig. 7). For $U_{a} \geq 0.35 \mathrm{~m} / \mathrm{s}$, passive walking or no walking behavior was observed. However, it was further observed that they could walk at any flow condition. This is because Zone $U$ (upstream of Block1) and Zone $B$ (gap between Block1 and Block2) provided good refugia. As a result, most of the invertebrates used active walking behavior for escaping out of the flume channel and most importantly no washout was observed for high flow conditions (Fig.8). However, the number of invertebrates remaining inside the Zone $B$ was higher compared to Zone $U$.

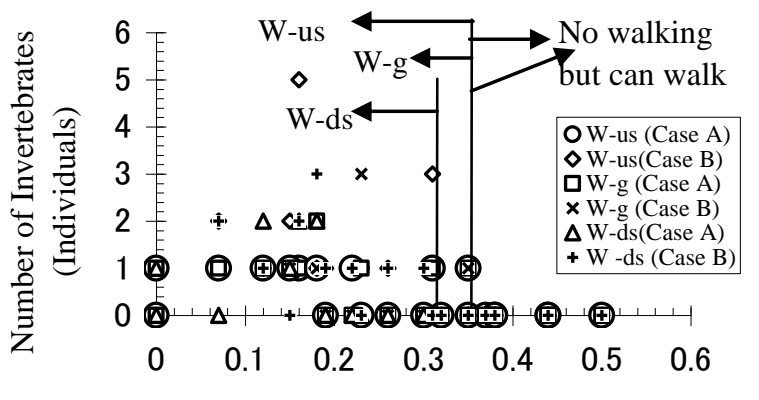

Depth averaged velocity, $U_{a},(\mathrm{~m} / \mathrm{s})$

Fig.7 Critical value of depth average velocity for no walking in Zone $U$ (W-us i.e. walking at upstream), Zone $B$ (W-g i.e. walking inside gap) and Zone $D$ (W-ds i.e. walking at downstream) -Case II (See Fig.2 and Fig.4)

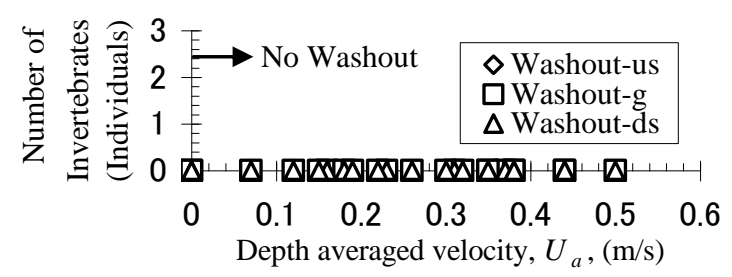

Fig.8 Critical value of depth-averaged velocity for washout in Zone $U$ (Washout-us i.e. washout at upstream), Zone $B$ (Washout - g i.e. washout inside gap) and Zone $D$ (washout $-\mathrm{ds}$ i.e. washout at downstream)-Case II (See Fig.2, Table 1 and Table 2) 
At Zone $D$, invertebrates mostly preferred central area (CA) and left wall region (LWR).The critical value of depth-averaged velocity for no walking in Zone $D$ is around $0.3 \mathrm{~m} / \mathrm{s}$ (Fig. 7). In this region also the invertebrates were not washed out of the flume in any flow condition (Fig. 8).

\section{DISCUSSION}

Irrespective of flow condition, without refugia, invertebrates endure all the time. However, endurance level depends on flow velocity. If the endurance level is low (small velocity i.e. $U_{a}$ less than $0.2 \mathrm{~m} / \mathrm{s}$, then invertebrates can walk very easily and actively. So, during passive walking stage (i.e. $U_{a}$ of $0.2-0.4 \mathrm{~m} / \mathrm{s}$ ), the force generated by flow is under their endurance limit. But, when the $U_{a}$ is more than $0.4 \mathrm{~m} / \mathrm{s}$, invertebrates are enduring the force more than they can tolerate. So, invertebrates are washed out. When refugia were introduced, such enduring behavior was not observed at all and as a result, they were not washed out or dislodged from its micro habitat.

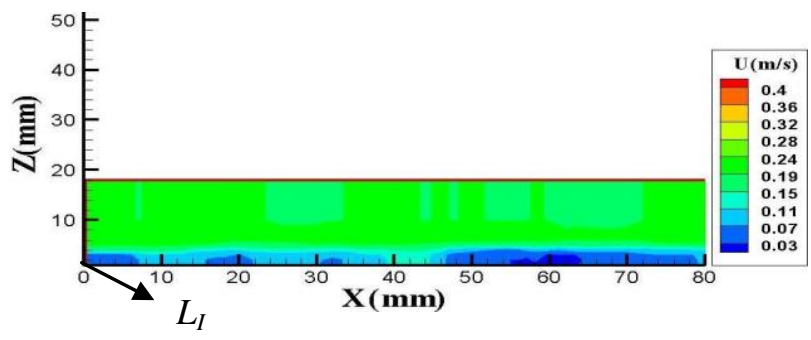

Fig.9 Velocity during low flow condition-no refugia- Case I (water depth $=1.8 \mathrm{~cm} \&$ depth-averaged velocity $=0.2 \mathrm{~m} / \mathrm{s}) ; L_{I}$ is the measurement position; Invertebrate height is $4 \mathrm{~mm}$.

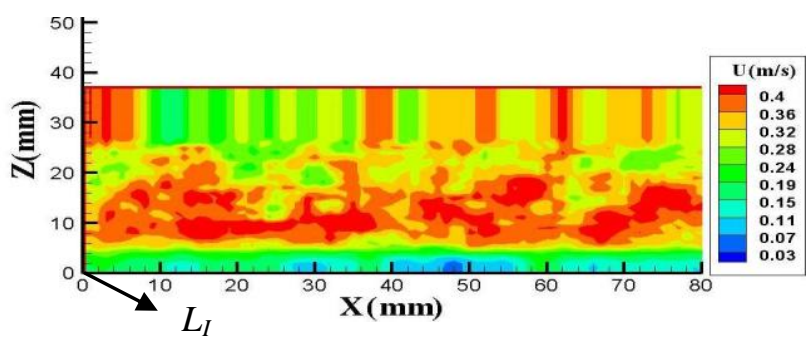

Fig.10 Velocity during high flow condition-no refugia- Case I (water depth $=3.8 \mathrm{~cm} \&$ depth-averaged velocity $=0.4 \mathrm{~m} / \mathrm{s}$ ); $L_{I}$ is the measurement position; Invertebrate height is $4 \mathrm{~mm}$.

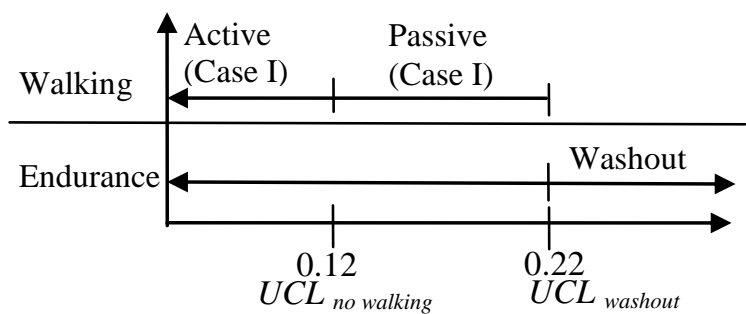

Fig.11 Summary for invertebrate's behavioral response to local velocity at invertebrate height: $U C L_{n o}$ walking means critical velocity for no walking and $U C L_{\text {washout }}$ means critical velocity for washout) -without refugia (Case I)
The local or regional velocity at the invertebrate height was analyzed from PIV experiment. The local velocity was calculated at $4 \mathrm{~mm}$ depth from the bottom (which is equal to the invertebrate height) for the corresponding critical depthaveraged velocity for no walking and washout $\left(U C_{n o}\right.$ walking \& $\left.U C_{\text {washout }}\right)$. The local critical velocity for no walking ( $U C L$ no walking $)$ and washout (UCL washout) is around $0.12 \mathrm{~m} / \mathrm{s}$ and $0.22 \mathrm{~m} / \mathrm{s}$ respectively (Figs.9, $10 \&$ 11).

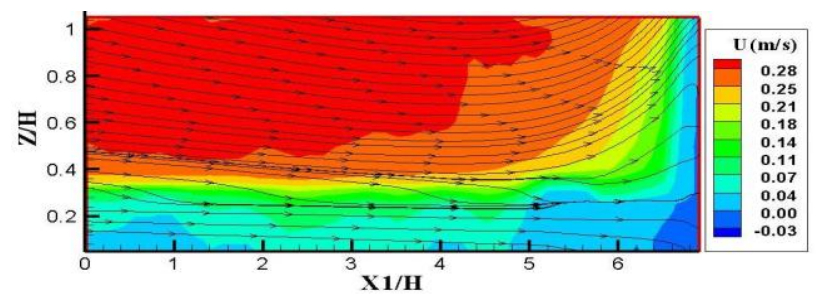

(a)

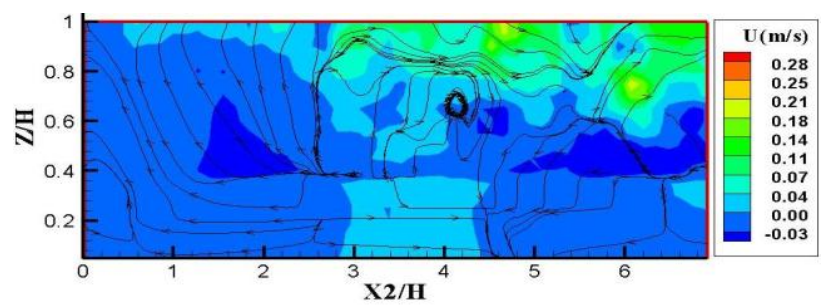

(b)

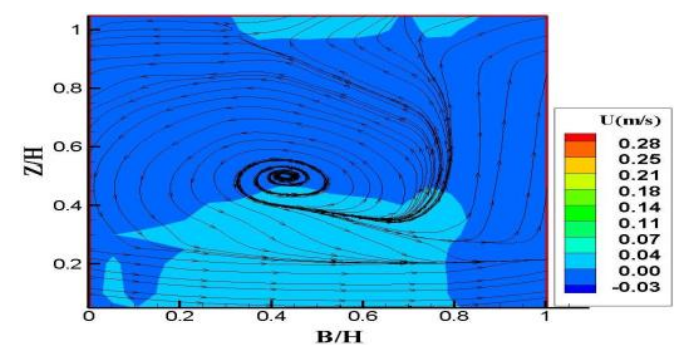

(c)

Fig. 12 Velocity and flow pattern (a) infront of Block1 as refugia (Zone $U$ ), (b) downstream of Block2 as refugia (Zone $D)$, (c) in between Block1 and Block2 as refugia (Zone $B$ ) during high flow condition $(7.4 \mathrm{~cm}$ water depth and $0.35 \mathrm{~m} / \mathrm{s}$ of depth average velocity); Invertebrate height is $Z / H$ of 0.2 .

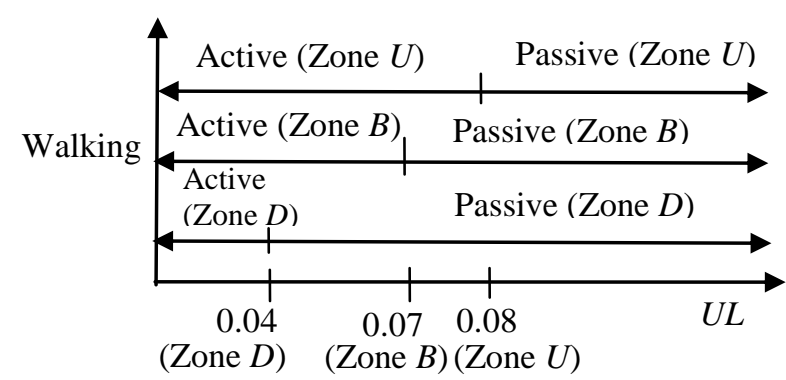

Fig.13 Summary for invertebrate's local velocity $(U L, \mathrm{~m} / \mathrm{s})$ around refugia (Case II) for a depth average velocity of $0.35 \mathrm{~m} / \mathrm{s}$

When refugia was introduced, the local velocity at the invertebrate height was always less than 0.1 $\mathrm{m} / \mathrm{s}$ at Zone $U$, Zone $B$ and Zone $D$ even during high 
flow condition (Figs. 12 \& 13). This value of local velocity with refugia is less than the no walking local critical velocity ( $U C L_{n o}$ walking of $0.12 \mathrm{~m} / \mathrm{s}$ ) without any refugia (Fig. 11). This is the reason why invertebrates don't get washed out or dislodged from their habitat during high flow conditions. This implies that refugia such as blocks can provide good shelter for invertebrates during flood conditions.

Bouckaert \& Davis ${ }^{7)}$ discussed about the benthic invertebrate's abundance in the wake than at the front boulders. However, in this study, it was also observed that the number of invertebrates remaining inside the Zone $B$ was higher compared to Zone $U$ and Zone $D$. One of the reasons could be the type of refugia used for the experiment. Our refugia (blocks) cover the whole width of the flume and flow from upstream overtops the blocks. So, the flow pattern would be different behind the refugia in our case than in the case of Bouckaert \& Davis ${ }^{7)}$. Fig. 12b shows the flow pattern behind the Block2. We can clearly see that for invertebrate height $(Z / H$ $=0.2$ ), flow pattern at downstream of Block2 is not complex. Moreover, an eddy is seen above the invertebrate height. As for the upstream region (Zone $U$ ), where some underscouring occurs beneath the Block1, no eddy is seen. Invertebrate can escape out of the flume by walking from upstream zone because in this region also, local velocity around the invertebrate height $(Z / H=0.2)$ is favorable for them to walk. In addition, swimming or drifting behavior was not observed at the upstream region. This can be explained by the fact that higher velocity exists from $Z / H \geq 0.4$ as shown in Fig 12a. We can see in Fig. 12c that inside Zone $B$, a large eddy is circulating in anti-clockwise direction just above the invertebrate height $(Z / H$ $=0.2$ ). In natural condition, this kind of arrangement not only provides a low flow habitat zone but rich food availability zone.

Moreover, the refugia setup used in this experiment is based on the study of Shah et al. ${ }^{8)}$. They concluded that the horizontal spacing of $B / H=1$ between two blocks is effective in reducing the drag and lift acting on the scoured frontal block and hence improves the stability of blocks. Our results show that this refugia setup is good for invertebrate's microhabitat from hydraulic point of view because it provides favorable velocity for invertebrates even during high flow conditions. So, for river ecosystem management, we can design the similar arrangement which can facilitate both stability and good refugia for invertebrate.

\section{CONCLUSION}

This experiment shows that an invertebrate behavior can be categorized based on the approach flow velocity without any refugia. They are active walking or crawling, passive walking or no walking, washout and enduring behavior. Invertebrates showed active walking behavior, for a depth average velocity less than $0.2 \mathrm{~m} / \mathrm{s}$, passive walking or no walking behavior for a depth average velocity of $0.2-0.4 \mathrm{~m} / \mathrm{s}$ and washout behavior for depth average velocity more than $0.4 \mathrm{~m} / \mathrm{s}$. Invertebrate showed endurance behavior from depth average velocity value of less than $0.4 \mathrm{~m} / \mathrm{s}$. The critical depth-averaged velocity for no walking is $0.2 \mathrm{~m} / \mathrm{s}$ and washout is $0.4 \mathrm{~m} / \mathrm{s}$. When refugia were provided, the invertebrates were able to walk for depth-averaged velocity of $0.35 \mathrm{~m} / \mathrm{s}$ i.e. is $40 \%$ increase in flow condition. With refugia, active walking and passive walking or no walking behavior was observed but washout behavior was not observed.

With no refugia, the critical local velocity around the invertebrate height for no walking is 0.12 $\mathrm{m} / \mathrm{s}$ and washout is $0.22 \mathrm{~m} / \mathrm{s}$. With the introduction of refugia, the local velocity around the invertebrate height was reduced to less than $0.1 \mathrm{~m} / \mathrm{s}$ for high flow conditions. Similar block arrangement (with horizontal spacing equal to height of the block) has a possibility to provide refugia in a stream.

ACKNOWLEDGMENT: The first author would gratefully like to acknowledge the Kyoritsu International Foundation for their financial support.

\section{REFERENCES}

1) Lancaster J.: Scaling the effects of predation and disturbance in a patchy environment, Oecologia, Vol. 107, pp. 321-331, 1996.

2) Dodds G.S. and Hisaw F.L.: Ecological studies of aquatic Insects: Adaptations of mayfly nymphs to swift streams, Ecological Society of America, Vol.5 (2), pp.137-148, 1924.

3) Matthaei C.D., Arbuckle C.J. \& Townsend C.R.: Stable surface stones as refugia for invertebrates during disturbance in a New Zealand stream, Journal of North American Benthological Society, Vol. 19(1), pp.82-93, 2000.

4) Lancaster J.: Small Scale movement of lotic macroinvertebrates with variations in flow, Freshwater Biology, Vol. 41, pp.605-619, 1999.

5) Cobb G.G., Galloway T.D. \& Flannagan J.F.: Effects of discharge and substrate on density and species composition of stream insects, Canadian Journal of Fisheries and Aquatic Sciences, Vol.49, pp.1788 -95, 1992.

6) Takemon, Y.:Emerging Behavior of Ephemera strigata and E. Japonica, Physiol.Ecol.Japan, Vol.22, pp.17-36, 1985.

7) Bouckaert F.W. \& Davis J.: Microflow regimes and the distribution of macroinvertebrates around stream boulders, Freshwater Biology, 1998, Vol. 40, pp.77-86, 1998.

8) Shah P., Tanaka N. \& Yagisawa J.: Effects of underscour depth and horizontal spacing between two bed protection blocks on stability of frontal block, Journal of Hydrodynamics, Vol.24(4), pp.579-588, 2012.

(Received September 30, 2012) 